\title{
Rodent posterior parietal cortex as a component of a cortical network mediating directed spatial attention
}

\author{
JAMES V. CORWIN \\ Northerm Illinois University, DeKalb, Illinois \\ and \\ ROGER L. REEP \\ University of Florida, Gainesville, Florida
}

\begin{abstract}
The rat posterior parietal cortex has been found to be a multimodal convergence area identifiable by its pattern of neuroanatomical connections with the cortex and thalamus. The rat posterior parietal cortex is part of a cortical network that also includes the medial agranular and ventrolateral orbital areas. Each of these three cortical areas is a region of multimodal convergence. Bilateral destruction of any of these areas produces deficits in spatial learning, whereas unilateral lesions induce deficits in directed attention. These findings suggest that together these cortical regions form an integrated network for spatial processing and orientation. In a recent test of this hypothesis, the posterior parietal area was selectively disconnected from the medial agranular cortex without direct damage to either area. This resulted in severe neglect which was qualitatively and quantitatively similar to that produced by unilateral destruction of wither cortical area, thereby supporting the network hypothesis. Some earlier conflicting reports bearing on the functional role of rat posterior parietal cortex may be explained in light of these disconnection data. The anatomical and behavioral findings suggest that in rats, as in primates, a parieto-frontal network mediates the integrated, dynamic spatial representations essential for normal directed attention and spatial orientation.
\end{abstract}

This review puts forward the viewpoint that the rodent posterior parietal cortex (PPC) is one component of a cortical network whose integrity is essential for the processing of multimodal information related to attention and spatial orientation. Support for this view is reviewed, focusing on (1) the findings of a number of anatomical studies that have demonstrated the existence of dense reciprocal connections among the PPC and other multimodal association areas, the medial agranular cortex $(\mathrm{AGm})$ and the ventrolateral orbital cortex (VLO); (2) behavioral and pharmacological studies that have shown dramatic similarities in the effects of lesions of these structures; and (3) the effects of disconnection of the PPC and AGm, which produces deficits that are similar to those produced by damage to either of these areas. In this review, we first present the anatomical relationships of the PPC, focusing on corticocortical and thalamocortical connections. Second, we discuss the central role of the PPC in attention, orientation, and spatial processing. Finally, we examine recent data that directly indicates that the PPC is part of a cortical network for directed attention and discuss

We thank Karen J. Burcham for her generous help with the figures and for reviewing earlier drafts of the manuscript. R.L.R. is in the Department of Physiological Sciences at the University of Florida. Correspondence concerning this article should be addressed to J. V. Corwin, Psychology Department, Northern Illinois University, DeKalb, IL 60115 (e-mail: tj0jvc1@corn.cso.niu.edu). the general implications of these findings for parietalprefrontal relationships.

\section{ANATOMY}

\section{Architecture}

Until recently, the existence of an anatomically defined PPC in rodents remained uncertain because of disagreements concerning parcellations made on architectural grounds. On the basis of cytoarchitectural and myeloarchitectural criteria adapted from Brodmann, Krieg (1946) identified a posterior parietal area 7 in the rat brain, located between the rostrally adjacent primary somatosensory area 2 and the caudally adjacent primary visual area 17 . Krieg found that, in comparison with area 2, area 7 had thin, compact layers II and III, a reduced but still distinct layer IV, and reduced pyramidal layers $\mathrm{Va}$ and $\mathrm{Vb}$. The secondary visual areas 18 and 18 a were pictured as forming part of a ring around area 17 , with area 18 as its medial component, area 18a located laterally, and area 7 as its rostral cap (Figure 1A). A similar scheme was delineated by Schober and Winkelmann (1975) for areas 17, 18, and 18a. Caviness (1975) renamed area 18 as area $18 \mathrm{~b}$ in his study of the mouse cortex, and this terminology was adapted to the rat by Vogt and Miller (1983). Although Rose (1912) had identified an area 7 in mice, Caviness (1975) felt that most of area 7 was actually part of area $18 \mathrm{a}$. Similarly, K. Zilles, B. Zilles, and Schleicher (1980) concluded that, 
A. Krieg (1946)
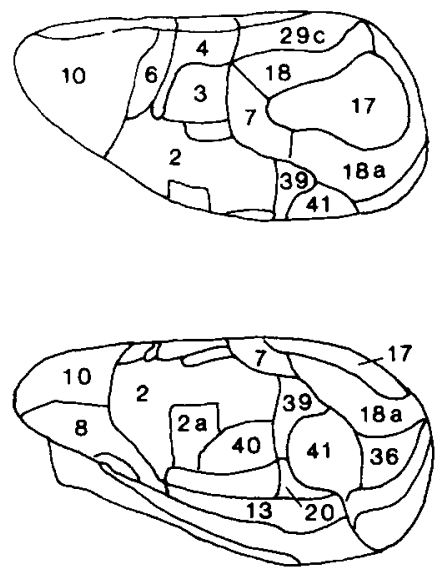

B. Zilles (composite)
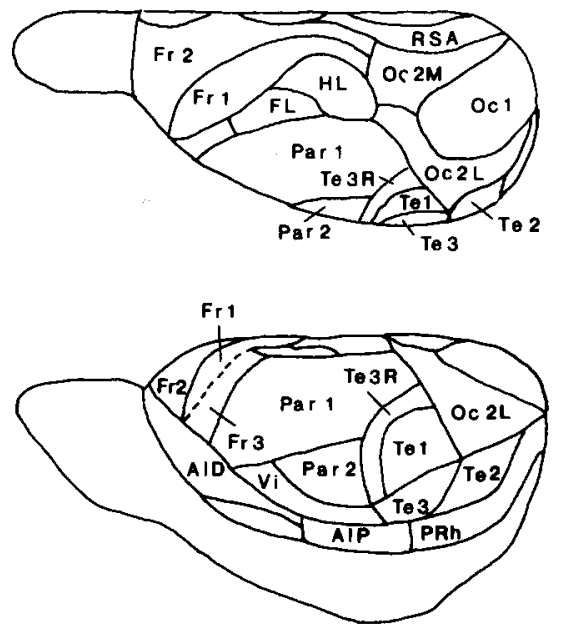

D.

Corwin \& Reep

(present report)
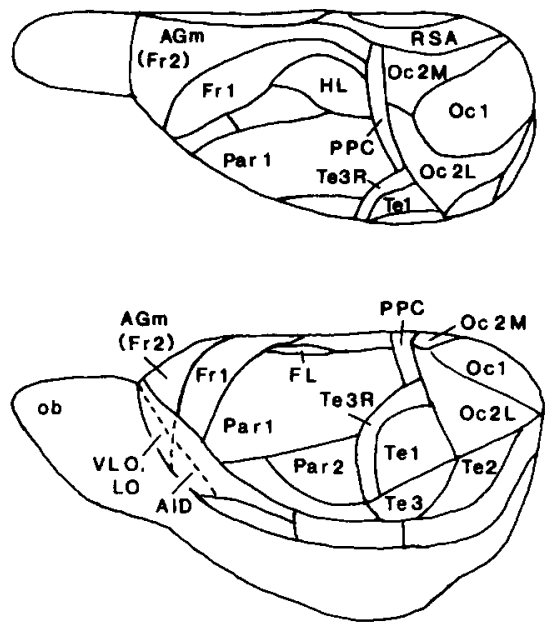

Figure 1. Four cortical maps of the rat brain. In each case, the top panel depicts a dorsal view, and the bottom panel presents a lateral view. (A) The early scheme of Krieg (1946) was based primarily on cytoarchitecture. (B) Composite map incorporating the work of Zilles et al. (1980), Zilles (1985), Zilles and Wree (1985) and Zilles et al. (1990), based on cytoarchitecture, myeloarchitecture, and chemoarchitecture. (C) Map of Vogt and Miller (1983), based on cytoarchitecture and connectivity patterns. (D) The present formulation, based on architecture and patterns of connections as delineated in Reep et al. (1990), Reep et al. (1994), and Reep et al. (1996). The dashed line in the bottom panel represents the unfolded rhinal fissure, in the depths of which lie the orbital areas frontally. Abbreviation: ob, olfactory bulb.

in rats, Krieg's area 7 actually belonged to area $18 \mathrm{a}$. Therefore, areas $18 \mathrm{a}$ and $18 \mathrm{~b}$ (their Oc2.1 and Oc2.2, which became Oc2L and Oc2M in the later formulation of K. Zilles and Wree [1985]) form a complete border around area 17 rostrally (Figure 1B). Vogt and Miller (1983) identified area 7 as lying rostral to a complete ring around area 17, formed by areas $18 \mathrm{a}$ and $18 \mathrm{~b}$ (Figure 1C). Thus, there developed agreement that areas $18 \mathrm{a}$ and $18 \mathrm{~b}$ formed a continuous border around area 17 rostrally, but disagreement remained concerning whether area 7 was wholly or partially subsumed by area $18 \mathrm{a}$. Kolb and Walkey (1987) felt that area 7 could be distinguished rostral to areas $18 \mathrm{a}$ and $18 \mathrm{~b}$, using the architectural criteria defined by Krieg. However, Chandler, King, Corwin, and Reep (1992) could find no consistent cytoarchitectural distinction between area 7 and the caudally adjacent areas 


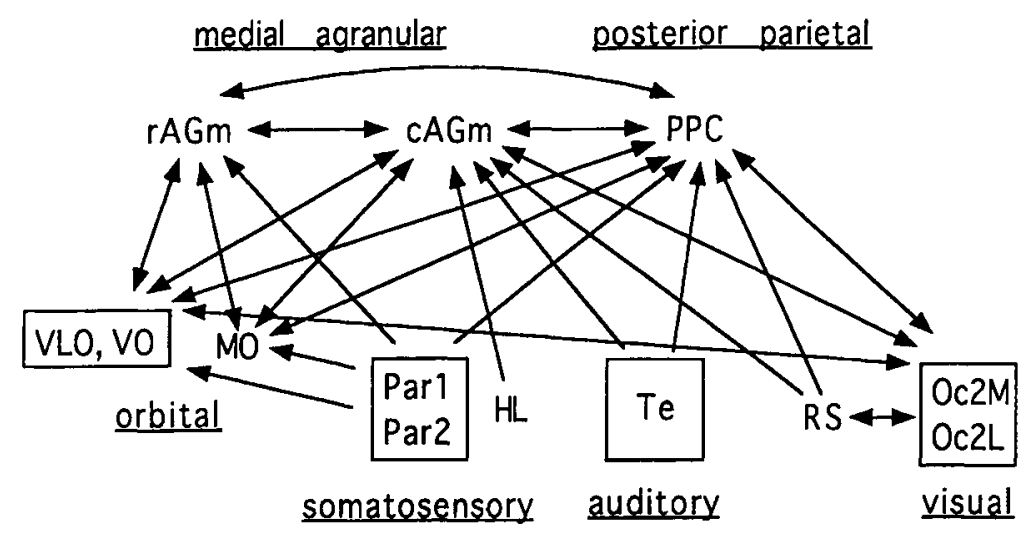

Figure 2. Schematic diagram of cortical connections involving the posterior parietal cortex, the medial agranular cortex, and the orbital cortex. Note the multimodal nature of the inputs reaching these three cortical regions and the interconnectivity among these three regions.

$18 \mathrm{a}$ and $18 \mathrm{~b}$. Each of these three areas possesses moderately developed layers IV and V, but the cell-sparse sublaminae of layer $\mathrm{V}$, which delineate its boundaries with layers IV and VI, are not as distinct as in primary sensory areas. Therefore, the cortical layers present a somewhat merged appearance. The rostral boundary of the PPC is readily distinguished from the somatic sensorimotor hindlimb area (HL), because of the latter's striking simultaneous presence of pronounced layers IV and $\mathrm{V}$, as well as a prominent cell-sparse zone in superficial layer $\mathrm{V}$ (Donoghue, Kerman, \& Ebner, 1979; Krieg, 1946; Reep, Chandler, \& Corwin, 1994; K. Zilles, 1985). Figure 1D presents the areal parcellation scheme and terminology employed by us, which derives largely from the formulation of K. Zilles. Krieg's area 7 is referred to as the PPC.

\section{Neuronal Connections of the PPC}

The limitations involved in the use of architectural criteria to define cortical areas can be overcome by comparing patterns of neuronal connections. The study of neuronal connections has helped to resolve the issues of whether there is a rodent PPC and of the extent of its boundaries. In any region, neighboring cortical areas may have significantly different connections that are correlated with functional specializations. Even if the PPC may not be distinguishable from the secondary visual areas on architectural grounds, its pattern of thalamocortical and corticocortical connections may differ.

Significant early observations concerning the PPC were made incidentally during the study of other cortical areas. Connections between area PPC and the visual cortex were first identified by Miller and Vogt (1984). They found that the PPC (their area 7) had reciprocal connections with areas $18 \mathrm{a}$ and $18 \mathrm{~b}$ and, less densely, with area 17. These findings were interpreted as suggesting that the PPC might represent a multimodal sensory convergence zone, though largely by analogy with other species, since, at the time, there was little evidence from rats to support this position.
In the early 1980 s, we began a series of anatomical studies on the connections of the AGm (equivalent to area Fr2 of $\mathrm{K}$. Zilles), in order to understand the means by which unilateral lesions of the AGm produced multimodal hemispatial neglect to visual, auditory, and somatic sensory stimuli. Leonard (1969) had suggested that this area was homologous to the frontal eye field (area 8) of primates, because both regions receive input from the thalamic mediodorsal nucleus and send projections to the superior colliculus. However, at this time, the cortical connections of the AGm were unknown.

In order to determine the cortical connections of area AGm, we first used retrograde axonal tracers, introduced into the AGm and surrounding areas. In these studies, we found consistent labeling in an area that we suspected might be the rodent PPC (Reep, Corwin, Hashimoto, \& Watson, 1984; see their Figures 3F-G). However, at that time, there was no firm justification for considering the PPC to be a distinct cortical area, and we included this labeling in the secondary visual areas (Oc2M and Oc2L), even though we recognized its more rostral location. Similarly, autoradiographic tracing of efferents from the AGm revealed labeling in the region of the PPC (Reep, Corwin, Hashimoto, \& Watson, 1987; see their Figures 3G-H).

The most striking finding in our studies of the AGm was the extensive number of cortical areas with which it was interconnected (Figure 2). Furthermore, the rostral and caudal portions of the AGm exhibit functional differences that are reflected in their patterns of connections with the somatic sensorimotor, visual, and retrosplenial cortices (Reep, Goodwin, \& Corwin, 1990). For example, rostral AGm receives extensive afferents from the caudal part of somatic sensorimotor area Par1, whereas caudal AGm receives input largely from the hindlimb cortex (area HL). Projections from the visual and retrosplenial cortices are much denser with caudal AGm than with rostral AGm. Afferents to the AGm from the orbital, perirhinal, and entorhinal cortices are all bilateral in origin and exhibit no topography within the AGm. The orbital con- 
nections involve the ventrolateral, ventral, and medial orbital areas.

These findings suggest that area AGm is a multimodal convergence zone. Furthermore, the AGm is connected with the PPC and the orbital cortex, which are also multimodal areas (discussed below). Area AGm is distinguished from the neighboring lateral agranular and anterior cingulate cortices by its having much more widespread cortical connections. The pattern of cortical connections of the AGm, together with its known subcortical connections (Leichnetz \& Gonzalo-Ruiz, 1987; Leichnetz, Hardy, \& Carruth, 1987; Miller, 1987; Neafsey \& Sievert, 1982; Reep et al., 1987) and functional properties (Donoghue \& Wise, 1982; Gioanni \& Lamarche, 1985; Hall \& Lindholm, 1974; Neafsey et al., 1986; Sanderson, Welker, \& Shambes, 1984; Sinnamon \& Charman, 1988; Sinnamon \& Galer, 1984), suggests that all of the AGm constitutes a frontal eye field that is involved in head orientation responses, with caudal AGm having more involvement in visual processing and rostral AGm directing motor output to the striatum and brainstem.

We were intrigued by the finding that the AGm had bilateral connections with the VLO. In the process of exploring the connections of the orbital fields, we were led once again to the PPC. Miller and Vogt (1984) had shown that the ventral and ventrolateral orbital areas (their area 11) were reciprocally connected with the secondary visual areas Oc2M and Oc2L. We began a behavioral study to test the hypothesis that unilateral lesions of the VLO would result in neglect to visual stimuli but not to somatic sensory or auditory stimuli. It soon became clear that neglect resulting from unilateral lesions of the VLO was multimodal rather than being limited to the visual modality (King, Corwin, \& Reep, 1989). This finding prompted us to examine the neuronal connections of the VLO and neighboring cortical areas, in order to determine the anatomical basis for this multimodal neglect.

Each of the four orbital areas has a distinct pattern of connections (Reep, Corwin, \& King, 1996). Corticocortical connections involving the ventrolateral and ventral orbital areas are more extensive than are those involving the medial and lateral orbital areas (Figure 2). Area VLO receives cortical input from the insular cortex, area $\mathrm{Fr} 2$, somatic sensory areas Parl and Par2, the PPC, and Oc2L. The ventral orbital area (VO) has connections with the cingulate area, the AGm, secondary somatic sensory area Par2, the PPC, and visual areas Oc2M and Oc2L. The medial orbital area (MO) has cortical connections with the cingulate cortex, the AGm, and the PPC. The lateral orbital area has cortical connections limited to the agranular and granular insular areas and Par2. These findings suggested that the extensive cortical connections of area VLO might underlie the multimodal neglect that is produced by unilateral lesions of it. It is interesting that areas MO and VO, like area VLO, have connections with the $\mathrm{AGm}$ and the PPC. This suggests the possibility that the $\mathrm{MO}$ and VO also play a role in directed attention and spatial processing. However, at present, there are no func- tional studies that have selectively examined the role of these areas in such processes.

Kolb and Walkey (1987) defined the rat PPC anatomically, on the basis of its connections, and reported that it was involved in allocentric spatial processes. Their anatomical results suggested that the rodent PPC could be identified by its thalamic input from the laterodorsal (LD) and lateral posterior (LP) nuclei, in the absence of afferents from the somatosensory ventrobasal nucleus (VB) or the visual dorsal lateral geniculate nucleus (DLG). In a systematic study of the afferent connections of the PPC and surrounding cortical areas, we demonstrated this to be the case (Chandler et al., 1992). Injections of retrograde axonal tracer into the PPC produce labeled neurons in the LD and LP and in the posterior nucleus, but none in the VB or DLG. The hindlimb cortex immediately rostral to the $P P C$ receives some input from the $L D$ and $L P$ but gets predominant input from the far lateral (hindlimb) portion of VB. The secondary visual areas Oc2M and Oc2L, situated caudal to the PPC, receive thalamic input from the LD and LP but also from the DLG. The medial boundary of the PPC abuts the retrosplenial cortex, which lies medial to the cingulum bundle and has some connections with the $L D$ and $L P$ but receives major input from the anterior nuclear group. The lateral boundary of the PPC abuts somatosensory area Par1, which receives a major input from the VB and has a distinctive cytoarchitecture that is characterized by a prominent layer IV and by well-defined cell-sparse sublaminae in layer V. Therefore, the PPC is distinguished by having dense input from the LD and LP exclusively, whereas the surrounding cortical areas have some input from the LD and LP, in addition to input from other thalamic nuclei. By these criteria of thalamic connectivity, the PPC is a thin, mediolaterally oriented area located at approximately AP -3.4 to $-4.4 \mathrm{~mm}$ relative to bregma and extending about $3 \mathrm{~mm}$ laterally from the cingulum bundle (ML 1.5-4.5; Paxinos \& Watson, 1986). This is smaller than the extent of the PPC suggested by Kolb and Walkey (AP - 4.0 to -6.0; ML 2.0-6.0) but corresponds well to the extent of area 7 delineated by Vogt and Miller (1983).

The cortical connections of the PPC also exhibit a pattern that is distinct from those of surrounding areas, although there are significant similarities between the cortical connections of the PPC and those of the secondary visual areas. Kolb and Walkey (1987) were the first to find that the PPC receives input from somatosensory area Parl, in addition to the visual cortex connections discovered earlier by Miller and Vogt (1984), which supports the view that the PPC represents a multimodal convergence zone. They also described connections between the PPC and the frontal multimodal convergence area AGm, consistent with our findings mentioned above. In experiments conducted by Reep et al. (1994), cortical injections of retrograde fluorescent tracers were utilized, and the PPC was defined on the basis of the thalamic criteria mentioned above. The cortical afferents of the PPC were found to involve the VLO and VO areas (note that the 
VO was not explicitly labeled in this report but was included in the MO area), the full extent of the AGm (Fr2), portions of somatic sensory areas Parl and Par2, secondary visual areas $\mathrm{Oc} 2 \mathrm{M}$ and $\mathrm{Oc} 2 \mathrm{~L}$, auditory area $\mathrm{Te} 1$, and the retrosplenial cortex (Figure 2). This pattern is easily distinguished from those of the neighboring somatic sensorimotor areas Parl and HL, which exhibit greatly reduced cortical connections in comparison with the PPC, and from that of the retrosplenial cortex, which has connections with the visual cortex, the caudal AGm, and the subiculum (Reep et al., 1994; Van Groen \& Wyss, 1992; Vogt \& Miller, 1983). The secondary visual areas Oc2L and Oc2M have cortical connections that are similar to those of the PPC, but, within the orbital cortex, they are focused in area VLO (Kolb \& Walkey, 1987; Miller \& Vogt, 1984; Reep et al., 1994; Vogt \& Miller, 1983), within the AGm they are restricted to its caudal portion (Miller \& Vogt, 1984; Reep et al., 1994; Reep et al., 1990; Sukekawa 1988; Torrealba, Olavarria, \& Carrasco, 1984; Vogt \& Miller, 1983), and they do not involve auditory area Te1. Further evidence that the PPC constitutes a distinct cortical area comes from recent detailed anatomical work on the connections of the somatosensory cortex. The area we have identified as the PPC corresponds to the parietal medial (PM) area, which has somatotopically organized connections with the somatosensory cortex. Neurons in the lateral PM project to the face area of primary somatosensory cortex (SI), whereas, in the medial PM, there is overlap in the projections to forelimb and hindlimb areas of SI (Fabri \& Burton, 1991; Koralek, Olavarria, \& Killackey, 1990). Neurons in the PM also project to the brainstem and spinal cord, as do neurons of the primary somatosensory cortex (Li, Florence, \& Kaas, 1990). Thus, the anatomical findings from several sources support the contention that the PPC is an anatomically distinct region in rodents.

\section{The PPC as a Multimodal Area}

The pattern of cortical connections of area PPC suggests that, like the AGm, it is a multimodal convergence zone, having connections with visual, auditory, and somatic sensorimotor areas. In addition, the PPC has reciprocal connections with the AGm and VLO, which are also multimodal areas. Electrophysiological evidence provides further support that the rat PPC has multimodal functions. Chen and McNaughton (1988) found that many neurons in the PPC exhibit movement selectivity and visual responses. McNaughton, Leonard, and Chen (1989) suggested that the combined coding of these properties in the PPC may be one component of a larger neural system that is concerned with spatial relationships and directed orientation. There is support for this in the recent finding by the same group that neurons selective for specific head directions are located in area Oc2M, apparently including the PPC (Chen, Lin, Green, Barnes, \& McNaughton, 1994).

The multimodal characteristics of area PPC are continued in the laterally contiguous cortical region, initially identified as area 39 by Krieg (1946). Neurons with combined auditory and somatosensory responses have been discovered in this region (Di, Brett, \& Barth, 1994). This area is located between the primary somatosensory and the auditory cortices and corresponds to architectural area Te3R of Zilles, Wree, and Dausch (1990), which is largely coextensive with area 39 of Krieg (1946). It forms a thin dorsoventral strip lying between the caudal boundary of somatosensory areas Parl and Par2 and the rostral boundary of primary auditory area Tel. This multimodal area receives predominant thalamic input from the posterior nucleus and the ventral subdivision of the medial geniculate nucleus, whereas the primary auditory area Tel gets afferents from the medial subdivision of the medial geniculate nucleus (Di et al., 1994; Roger \& Arnault, 1989; Romanski \& LeDoux, 1993). In another study, injections in what appears to be the same region produced labeling in the posterior nucleus and medial subdivision of the ventral posterior nucleus but not in the medial geniculate nucleus (Arnault \& Roger, 1990). Nothing is known about the behavioral consequences of lesions restricted to this somatosensory-auditory area.

\section{Comparison With the Monkey PPC}

The patterns of connectivity described above for the rat PPC are similar in certain respects to patterns seen in the primate PPC. In rhesus monkeys, area 7 contains several subfields identifiable on the basis of cytoarchitecture and connections (Cavada \& Goldman-Rakic, 1989a; Schmahmann \& Pandya, 1990). Thalamic afferents to all portions of monkey area 7 originate mostly from the pulvinar but also from the LP (Schmahmann \& Pandya, 1990). Although rats have no pulvinar, the existence of an LPpulvinar complex is recognized across mammals, and it is likely that the LP is the homologous structure in species lacking a pulvinar (Harting, Hall, \& Diamond, 1972). Therefore, we conclude that the rat PPC receives thalamic input from a source similar to that supplying area 7 in monkeys.

In rhesus monkeys, the PPC has extensive reciprocal connections with the frontal and orbital cortex (Cavada \& Goldman-Rakic, 1989b), and the connections between the frontal cortex and the PPC are denser than those between any other association areas in the primate brain (Selemon \& Goldman-Rakic, 1988). Similarly, in the rat, there are extensive reciprocal connections among the medial agranular, orbital, and posterior parietal cortices (Reep et al., 1994; Reep et al., 1984, 1987; Reep et al., 1996; Reep et al., 1990). In monkeys, visual connections predominate in the caudal area $7 \mathrm{a}$, whereas the more rostral area $7 \mathrm{~b}$ communicates with somatosensory fields (Cavada \& Goldman-Rakic, 1989a). No such modalityspecific topography has been identified within the rat PPC.

In rats, cortical areas AGm, VLO, and PPC share the attributes of having multimodal connections and being interconnected among themselves. Furthermore, as in primates, these areas are involved in directed attention and spatial behavior, as described below. Therefore, we 
hypothesize that the AGm, VLO, and PPC form a cortical network for spatial attention and spatial learning, similar to that suggested for the monkey by Selemon and Goldman-Rakic (1988).

\section{Interconnections Among the PPC, AGm, and VLO}

Behavioral studies typically utilize large lesions that affect several cortical areas and often affect the underlying white matter as well. The results of such studies are difficult to interpret in the context of neuronal circuitry, because of large interanimal variability in the anatomically defined regions that are damaged and because fibers of passage are affected with white matter damage. For these reasons, we have focused on the functional roles of the AGm, PPC, and VLO by performing behavioral experiments after making lesions in specific cortical areas defined by their patterns of connections. In this sense, our understanding of the neuroanatomical organization has provided a guide for the behavioral work.

Our interest in the PPC and VLO developed initially out of the discovery that these areas are interconnected with the AGm. The results of experimental studies performed over the past 15 years have shown that each of these areas has the anatomical and functional properties of a multimodal association cortex. Because these cortical areas are likely to function as a network, as we will argue in the behavioral review section, it is instructive to consider the pathways by which they are interconnected.

In earlier reports, we described autoradiographic labeling in rats that suggested that the projection from area AGm to visual association areas travels in the deep gray matter, rather than in the white matter (Reep et al., 1987; Reep \& Goodwin, 1988). We also noted that, in dorsally situated cortical areas, layer VII, the deepest cell stratum, is separated from the overlying layer VI by a cell-sparse, axon-rich zone in which many of these axons appear to travel (Reep \& Goodwin, 1988). In a recent study (Vandevelde, Duckworth, \& Reep, 1996), the longitudinally directed axons from the AGm to the PPC and visual cortex were directly visualized, using the fluorescent axonal tracer fluororuby and sagittal sections. Most of these corticocortical axons travel in the deep gray matter, whereas axons destined for subcortical targets travel in the underlying white matter. The corticocortical axons aggregate most densely in the cell-sparse zone superficial to layer VII, but many travel in layer VI as well. Axons traveling to the AGm from caudal cortical areas like Oc2M and PPC utilize a similar trajectory. Furthermore, intrahemispheric axons traveling in the coronal rather than the sagittal plane also travel predominately in the deep gray matter.

These findings hold significant implications for the interpretation of behavioral findings involving non-axonsparing lesions, as in most of the PPC literature. Any cortical lesion extending into layer VI would not only have destroyed tissue in the area lesioned but would have also transected axons passing through the area, even if it did not effect the white matter.
The finding that corticocortical axons travel in the deep gray matter presented the opportunity to test the hypothesis that the AGm, PPC, and VLO function as a distributed cortical network for directed attention. If intact circuitry linking these three areas is required for normal directed attention, disruption of these connections in the absence of damage to any of the areas themselves should produce neglect. We reasoned that, because of their longitudinal trajectories, the axons linking the AGm and the PPC could be selectively transected by making a coronally oriented knife cut, without disrupting other axons traveling in the deeper white matter and without causing major damage to the cortical area in which the knife cut was made. Studies directly examining this prediction will be discussed in subsequent sections of this paper.

\section{ROLE OF THE RODENT PPC IN SPATIAL LEARNING}

The results of anatomical studies of the PPC suggest that, as was found for the PPC in primates, rodent PPC is a multimodal convergence area that is interconnected with other cortical regions receiving multimodal inputs. These findings strongly suggest that the PPC may function as one component of a distributed cortical system. In this section, we will address some of the behavioral findings on the cortex and spatial processing in rodents, to determine whether the results of these studies support the system perspective (Goldman-Rakic, 1988; Heilman, Watson, \& Valenstein, 1985; Mesulam, 1990). We will argue that considering the PPC to be one component of this system may provide a new perspective on the parietal and prefrontal literature in rodents. Further, the anatomy of the rodent cortex provides a unique model for testing several hypotheses generated by this system approach.

\section{Effects of the PPC Lesions on Spatial Learning}

The role of the PPC in spatial behavior and the cognitive representation of space has been well documented in primates. A number of studies have indicated that humans with PPC damage demonstrate a variety of spatial deficits. They demonstrate an inability to follow maps of external space (Semmes, Weinstein, Ghent, \& Teuber, 1963), deficits in internal representations of space, as in hemispatial neglect (Bisiach \& Luzzati, 1978; Heilman, Watson, \& Valenstein, 1993), and disorders of block construction or drawing of objects (Paterson \& Zangwill, 1944). Parietal destruction in nonhuman primates also produces spatial impairments on landmark reversal tasks (Pohl, 1973), disabilities in cage finding (Sugishita, Ettlinger, \& Ridley, 1978), and neglect (Deuel, 1987).

Although there is a long history that chronicles the involvement of the PPC in spatial behavior, it is only in the last 15 years that this issue has been examined with regard to the rodent PPC. An interest in the role of the rodent PPC in spatial processing grew out of several basic findings. First, several researchers had clearly established that rodents possessed an anatomically distinct pre- 


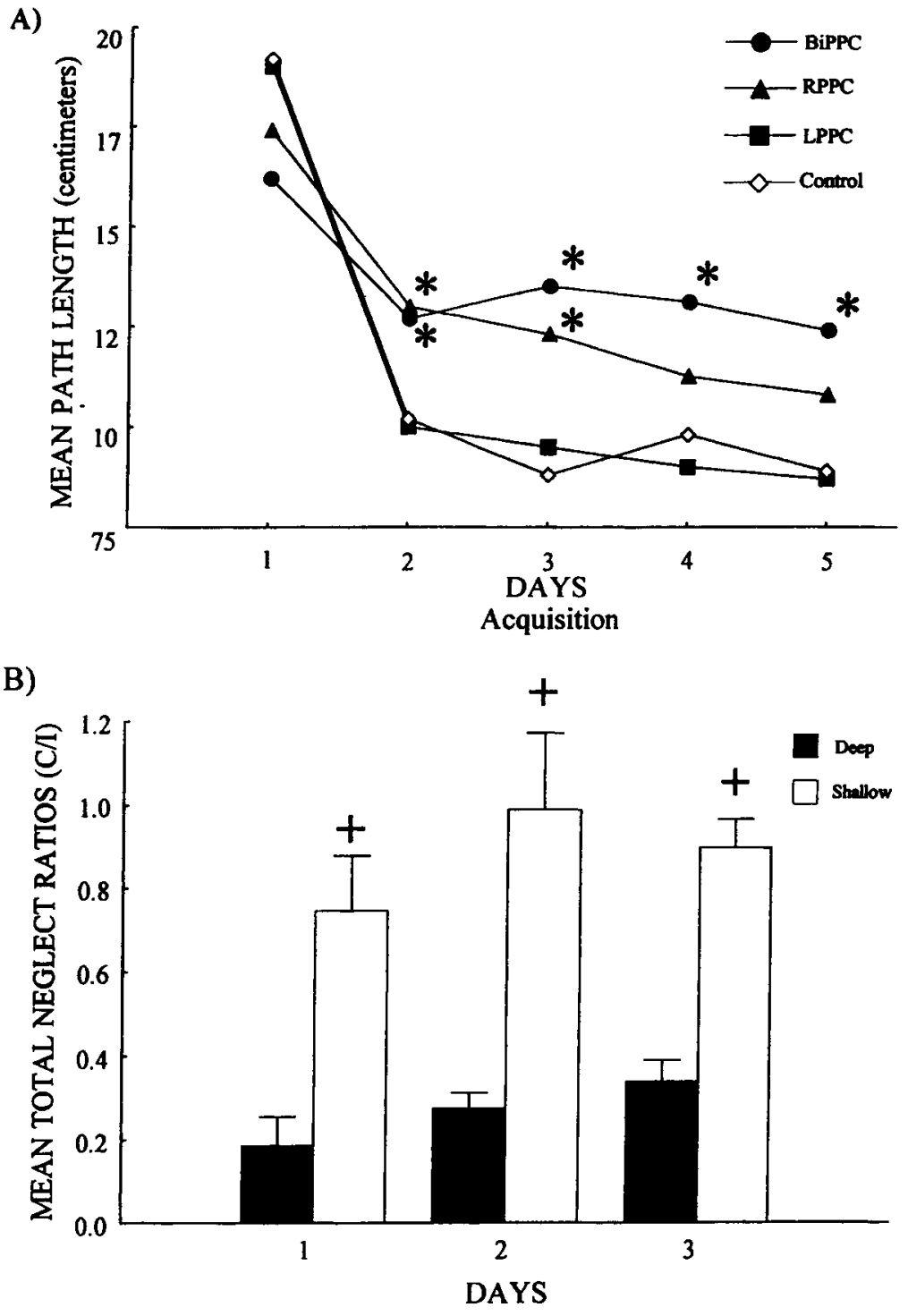

Figure 3. (A) The effects of bilateral (BiPPC), unilateral right (RPPC), and unilateral left (LPPC) posterior parietal cortex lesions relative to control operates in a cheese board allocentric spatial task. An asterisk indicates a significant difference from the control and LPPC groups. From "Spatial Deficits and Hemispheric Asymmetries in the Rat Following Unilateral and Bilateral Lesions of Posterior Parietal or Medial Agranular Cortex," by V. King and J. V. Corwin, 1992, Behavioural Brain Research, 50, p. 62. Copyright 1992 by Elsevier Science-NL. Adapted with permission. (B) Total neglect ratios for the deep and shallow knife-cut groups. Total neglect ratios were obtained by summing across the visual, tactile, and auditory stimulus modalities for the contralesional and ipsilesional body sides and forming a ratio of contralesional/ipsilesional responsiveness (C/I). A smaller neglect ratio indicates more severe neglect, whereas a ratio of 1.0 indicates symmetrical responsiveness. From "Spatial Deficits and Hemispheric Asymmetries in the Rat Following Unilateral and Bilateral Lesions of Posterior Parietal or Medial Agranular Cortex," by V. King and J. V. Corwin, 1992, Behavioural Brain Research, 50, p. 62, and from "Disconnection of Medial Granular and Posterior Parietal Cortex Produces Multimodal Neglect in Rats," by K. J. Burcham, J. V. Corwin, M. L. Stoll, and R. L. Reep, 1997, Behavioural Brain Research, 86, p. 43. Copyright 1992, 1997 by Elsevier Science-NL. Reprinted with permission. 
frontal association cortex located on the pregenual medial wall and rhinal fissure (Krettek \& Price, 1977; Leonard, 1969,1972 ) that appeared to function qualitatively like the primate prefrontal cortex (Kolb, 1984). Further, lesions of the rodent prefrontal cortex produced deficits in spatial processing and in the sequencing of behavior (Kolb, 1984; Kolb, Nonneman, \& Singh, 1974; Nonneman, Voigt, \& Kolb, 1974). Second, behavioral tasks had been developed that allowed for an examination of the use of specific spatial strategies in rodents-most importantly, the radialarm maze (Olton, 1977) and the water maze (Morris, Garrud, Rawlins, \& O'Keefe, 1982). The finding that rodents possessed a complex prefrontal cortex that was qualitatively similar both anatomically and functionally to primates strongly suggested that a PPC must also be present, and the new behavioral technologies allowed for the possibility of testing for the effects of the anticipated spatial deficits resulting from lesions of the presumed PPC.

The earliest behavioral studies of the rodent PPC directly compared the effects of PPC lesions with those resulting from medial prefrontal and sulcal prefrontal lesions, and from complete decortication in several spatial tasks, including the radial-arm maze and water maze (Kolb, Sutherland, \& Whishaw, 1983). The results of these studies indicated that the decorticates and the medial prefrontal and sulcal prefrontal operates demonstrated egocentric and allocentric spatial deficits. However, by comparison, destruction of the PPC, as defined by using the projection field of thalamic nuclei LD and LP (as defined by case R12 in the study by Jones \& Leavitt, 1974), resulted in rather mild allocentric spatial deficits. Thus, unlike the devastating effects of PPC lesions in primates, only moderate deficits were found in some aspects of spatial performance, and the effects of the PPC lesions were mild, compared with the more severe deficits caused by medial frontal lesions, sulcal prefrontal lesions, or complete decortication. The authors concluded that the PPC in rodents was less involved in spatial learning than was the prefrontal cortex and hippocampus (Kolb et al., 1983).

In a subsequent study, Kolb and Walkey (1987) reexamined both the anatomy and function of the rodent PPC. The anatomical results suggested that the PPC could be identified as a distinct region receiving thalamic afferents from the LP and LD. Further, lesions of this region produced spatial deficits, relative to controls in the radial-arm maze, and deficits in place and landmark navigation in a water maze. These deficits were more severe, relative to controls, than those reported in the Kolb et al. (1983) study. A comparison of the extent of the lesions in the two studies is difficult, but it would appear that the lesions extended farther caudal in the Kolb and Walkey study and more completely damaged the PPC. The results of the Kolb and Walkey study, when combined with the findings of Kolb et al. (1983), suggested that two regions of the prefrontal cortex (medial and sulcal), as well as the PPC, are involved with allocentric spatial processing. However, the circuitry that would underlie the involvement of these areas in allocentric spatial pro- cessing was unknown. Kolb and Walkey found that the PPC had some connections to the prefrontal cortex, the dorsal anterior cingulate, and the AGm. Projections from the orbital cortex were found but were believed to result from labeling by uptake from fibers of passage traveling to the ventral orbital region.

A series of investigations of the parietal and prefrontal cortices were done by Kesner and his colleagues in order to further delineate the functions of these areas in spatial processing (DiMattia \& Kesner, 1988a, 1988b; Kesner, Farnsworth, \& DiMattia, 1989). The prefrontal lesions in the Kesner studies (Kesner et al., 1989) mainly damaged the rostral AGm and the dorsal anterior cingulate cortex. A classic double-dissociation was found. The prefrontal operates demonstrated a deficit in egocentric spatial processing in an adjacent-arm maze task but no deficit for allocentric spatial orientation in a water maze. The behavioral findings in their prefrontal subjects were in contrast to those of Kolb et al. (1983), in which animals with more extensive medial prefrontal destruction demonstrated both egocentric and allocentric spatial deficits. Further, their PPC lesions produced severe deficits in allocentric spatial tasks (cheese board, radial-arm maze, and water maze), unlike the Kolb et al. findings and in support of the results of Kolb and Walkey (1987). The allocentric deficits demonstrated by the PPC operates were more severe than those exhibited by animals with hippocampal lesions (DiMattia \& Kesner, 1988b). An examination of the parietal lesions in the Kesner et al. studies indicated that their PPC lesions extended from $0.5 \mathrm{~mm}$ rostral to bregma to $3.5 \mathrm{~mm}$ caudal to bregma and from $2 \mathrm{~mm}$ lateral to the sagittal suture to the rhinal fissure. The extent of the parietal lesions, as described and as indicated in their histology figures, was rostral to the area that is currently considered to be the rodent PPC (Kolb \& Walkey, 1987; Reep et al., 1994) and also would have encompassed a number of other cortical areas, including Parl, Par2, and HL. Thus, a large parietal cortex lesion, but without direct PPC involvement, was able to produce dramatic deficits in allocentric spatial learning. However, the large medial-lateral extent of the lesions was likely to have destroyed many of the rostrally projecting efferents of the PPC traveling in layer VI of the cortex (Vandevelde et al., 1996).

Two subsequent studies examined the effects of unilateral destruction of the PPC (Crowne, Novotny, Maier, \& Vitols, 1992; King \& Corwin, 1992). Both studies found that unilateral right-hemisphere lesions or bilateral PPC lesions produced deficits in allocentric spatial tasks, whereas left-hemisphere PPC lesions did not (Figure 3A). In the King and Corwin (1992) study, the lesions extended caudally to encompass the regions that were suggested by Kolb and Walkey (1987) to be the rodent PPC (2-6 mm posterior to bregma, 3-6 mm lateral to the sagittal suture). The lesions in the Crowne et al. (1992) study were even more extensive but were intended to destroy the PPC. The results of the King and Corwin (1992) study replicated the double dissociation between the prefrontal cortex and 
the PPC found by Kesner and his colleagues and found that prefrontal lesions restricted to the AGm would produce the prefrontal egocentric deficit. Another recent examination of the effects of unilateral PPC lesions also found hemispheric asymmetries in PPC function (McDaniel et al., 1995). In contrast to prior studies (Crowne et al., 1992; Kesner et al., 1989; King \& Corwin, 1992; Kolb, Buhrmann, McDonald, \& Sutherland, 1994), they reported that the major effect of PPC lesions was on egocentric spatial learning, as assessed by response learning in a Greek cross maze. However, their PPC lesions infringed on both the anterior PPC and the caudal AGm. King and Corwin found that AGm lesions can produce egocentric spatial deficits, but, in that study, the effect of unilateral AGm destruction on egocentric spatial processing was not found to be lateralized, and the lesions included the entire AGm (King \& Corwin, 1992). Therefore, the interpretation that the PPC is involved in egocentric spatial performance cannot be ruled out at this time, but the interpretation is somewhat clouded by the additional involvement of caudal AGm in the PPC lesions. However, more recently, Save and Moghaddam (1996) have reconciled these findings. They found that bilateral PPC lesions (associative parietal cortex, in their terminology) produced deficits in both egocentric and allocentric learning situations. They suggested that the PPC plays the role of an interface between egocentric and allocentric learning by constantly integrating kinesthetic information on egocentric head position with visual information on allocentric stimuli, as suggested by Chen et al. (1994) and Kolb et al. (1994).

\section{The PPC as One Component of a Cortical Network}

The above review indicates that there were discrepancies in the literature concerning the roles of the PPC and the prefrontal cortex in spatial processing in rodents. Several studies had indicated that the PPC was involved with allocentric spatial processing, but this was not different from the deficit after large medial frontal or orbital prefrontal lesions (Crowne et al., 1992; Kesner et al., 1989; King \& Corwin, 1992; Kolb et al., 1983; Kolb \& Walkey, 1987). Studies in which the lesions did not directly damage what is currently considered the PPC produced allocentric spatial deficits that were more severe than hippocampal operates (DiMattia \& Kesner, 1988b; Kesner et al., 1989) and that were virtually identical to the deficits obtained in studies that directly damaged the PPC (Crowne et al., 1992; King \& Corwin, 1992; Kolb \& Walkey, 1987). Also, large prefrontal lesions produced both egocentric and allocentric deficits, but, with more restricted lesions of the prefrontal cortex, involving just the $\mathrm{AGm}$, only egocentric spatial deficits were obtained (Kesner et al., 1989; King $\&$ Corwin, 1992). Given these findings, it was unclear whether there was a well-defined cortical system for spatial processing in rodents, as had been suggested for primates (Selemon \& Goldman-Rakic, 1988).

A series of recent studies directly compared the effects of rostral parietal lesions, using the lesion coordinates of
Kesner and his colleagues, with the effects of more caudal lesions that directly involved the PPC, using the coordinates of Kolb and Walkey (1987; Save, Poucet, Foreman, \& Buhot, 1992; Thinus-Blanc, Save, Poucet, \& Foreman, 1996). Both lesion groups were tested in an allocentric task (three-table maze), and no differences between the groups were found (Thinus-Blanc et al., 1996). Both the anterior and the posterior parietals were impaired on the early trials, although not to the degree found in complete medial prefrontal operates in a prior study (Herrmann, Poucet, \& Ellen, 1985). In a habituation task (Save et al., 1992), anterior parietal cortex and hippocampal operates showed a significant increase in exploratory behavior when objects in a previously explored environment were changed spatially, whereas PPC and hippocampal operates did not respond to the change. When a novel object was introduced, only PPC operates did not respond to the change with increased exploration. However, there were no significant differences between the anterior and posterior parietal groups on any of the measures. The results of these studies supported the findings of both Kesner et al. (1989) and Kolb and Walkey, in that anterior parietal lesions, as well as PPC lesions, produced allocentric spatial deficits.

An examination of the connections of the PPC held a clue to integrating some of these discrepancies in the literature. Chandler et al. (1992) and Reep et al. (1994) found that the PPC of rodents was very strongly interconnected with two cortical regions, the VLO and the AGm. Several studies had indicated that unilateral destruction of any one of these three regions produced severe multimodal neglect (Corwin et al., 1986; Crowne \& Pathria, 1982; Crowne, Richardson, \& Dawson, 1986; King \& Corwin, 1993; King et al., 1989). Although the effects of PPC and AGm destruction on spatial processing were known, the effects of VLO destruction on spatial learning had not been determined. This issue had some relevance to a better understanding of the effects of large prefrontal surgeries (Kolb et al., 1994; Kolb et al., 1983), as well as to studies that produced PPC-like deficits but did not directly damage the PPC (DiMattia \& Kesner, 1988a, 1988b; Kesner et al., 1989; the anterior parietal groups in Save et al., 1992; and Thinus-Blanc et al., 1996).

Corwin et al. (1994) examined the role of the VLO in egocentric and allocentric spatial processing, using the adjacent-arm maze task as an egocentric task and the cheese board task as an allocentric spatial task (Kesner et al., 1989). Bilateral destruction of the VLO but not of the laterally adjacent lateral orbital cortex resulted in a significant deficit in the cheese board task, but not in the adjacent-arm maze task. The finding that the VLO is involved in allocentric spatial processing may explain why the large medial frontal and orbital lesions in the Kolb et al. (1983) study produced such profound allocentric spatial deficits. It is likely that these lesions disrupted the connections from the PPC to the VLO, as well as producing direct orbital damage. Likewise, it is likely that the large parietal lesions of Kesner et al. and the anterior parietal lesions of Thinus-Blanc et al. (1996) destroyed the con- 
nections from the PPC to the VLO. The representative lesions presented in both of these studies clearly extended down to and apparently through the white matter in some cases. Therefore, it is likely that the anterior lesions produced destruction of many of the rostrally projecting efferents of the PPC that travel in layer VI (Burcham, Corwin, Stoll, \& Reep, 1997; Vandevelde et al., 1996). The results of these studies are consistent with a potential disconnection effect in the anterior parietal group and with direct damage in the PPC group.

The anatomical interconnectivity among the PPC, the $\mathrm{AGm}$, and the VLO and the behavioral results of destruction of these areas on spatial processing suggest that these areas are components of an interconnected cortical network for spatial processing in rodents. This conception places much of the rodent literature on spatial learning into an anatomical context. The converging multimodal sensory connections of the PPC, AGm, and VLO place these regions in the forefront for the processing of external stimuli in space and for the movement of the organism in space during egocentric and allocentric spatial tasks. A crucial issue that remains to be addressed is whether these regions (the PPC, AGm, and VLO) function in parallel or alternatively as an integrated system for spatial processing. Clearly, disconnection studies are required as a direct test of whether these regions function as an integrated circuit for the processing of spatial information. As will be discussed in the next section, such disconnection studies are feasible in rodents (Burcham, Corwin, et al., 1997; Burcham, Corwin, \& Reep, 1997), because the trajectories of the fiber connections among these structures travel in layer VI of the cortex (Vandevelde et al., 1996). However, this issue remains unresolved with regard to spatial-learning tasks.

\section{ROLE OF THE RODENT PPC IN DIRECTED ATTENTION}

In addition to its involvement in spatial learning, the rodent PPC has been found to play a role in directed attention and orientation. Evidence will be presented supporting the contention that the PPC functions as an important component of an interconnected cortical network for directed attention that involves three cortical regions: the PPC, the AGm, and the VLO.

\section{Effects of Unilateral PPC Destruction in Primates}

Patients sustaining inferior parietal lobule damage in the area of the right parieto-temporal-occipital junction demonstrate a remarkable deficit in directed attention that is characterized by a failure to report, respond, or orient to novel or meaningful stimuli presented to the side of the body opposite a lesion (Heilman et al., 1993). In the most severe cases, these patients shave only the right (ipsilesional) side of their face, dress only the right side of the body, demonstrate inappropriate orientations by responding to contralesional stimulation as if it came from the ipsilesional side (allesthesia/allokinesia), and show a general paucity of movement in the contralesional side of space. The deficit is not just a lack of responsiveness to sensory stimulation but also a deficit in the cognitive representation of space. When asked to describe a mental representation of a well-known scene, neglect patients describe objects only in the right side of space. When asked to reverse the spatial perspective, they now describe objects on the right side of the reversed perspective and cannot describe the previously described objects (Bisiach \& Luzzati, 1978). The deficit is clearly extraordinarily complex and spans attentional, spatial, sensory, and motor behavior (Heilman et al., 1993). It has been estimated that some manifestation of neglect is found in $40 \%-60 \%$ of all cases of right-hemisphere damage, and the vast majority of all cases of neglect are induced by cortical damage (Heilman et al., 1985).

In humans, the PPC has been the area most often implicated in attention and deficits in attention, with the most severe deficits resulting from destruction of the cortex in the parieto-temporal-occipital junction (Heilman et al., 1993; Vallar, 1993). However, the deficit is found not only in the case of parietal lobule damage but also following destruction of the anterior cingulate or dorsolateral prefrontal cortex (area 8) (Heilman et al., 1985). The nature of the deficits following destruction of any one of these three cortical regions is remarkably similar. This finding has prompted several investigators to suggest that these three regions (the PPC, the cingulate cortex, and area 8) form a cortical circuit for directed attention in space (Goldman-Rakic, 1988; Heilman et al., 1985; Mesulam, 1990). A similar corticocortical circuit has been suggested for nonhuman primates. In monkeys, neglect is induced by unilateral destruction of the PPC, the dorsolateral prefrontal, or the cingulate cortex (Heilman et al., 1993). However, the most severe deficits are typically produced by destruction of area 8 (Deuel, 1987).

\section{Effects of Unilateral PPC Destruction in Rats}

In rodents, the early work on cortically induced neglect did not focus on the role of the PPC but rather on the dorsomedial prefrontal cortex, which includes the AGm, the rodent counterpart of area 8 in primates. As in humans and monkeys with neglect, rats sustaining unilateral lesions of the dorsomedial prefrontal cortex show a dramatic deficit in the ability to orient to simple visual, tactile, auditory, and nociceptive stimuli (Cowey \& Bozek, 1974; Crowne, 1983; Crowne \& Pathria, 1982). In addition, these animals demonstrate allesthetic/allokinetic responses, wherein animals orient to contralesionally presented stimuli by turning toward the ipsilesional side. Later anatomical (Reep et al., 1984, 1987; Reep et al., 1990) and behavioral (Corwin et al., 1986; King \& Corwin, 1990; Vargo, Corwin, King, \& Reep, 1988) studies indicated that area AGm was the cortical area that was crucial for the production of severe neglect.

As discussed previously, early anatomical studies of area AGm, the rodent analog of the frontal eye field, re- 
vealed interconnections among the AGm, the VLO, and an area initially considered as a part of area Oc2M, later identified as the PPC (Chandler et al., 1992; Kolb \& Walkey, 1987; Reep et al., 1994; Reep et al., 1984, 1987; Reep et al., 1996; Reep et al., 1990). The striking interconnectivity of these regions and the dramatic multimodal neglect produced by unilateral lesions restricted to the AGm suggested the possibility that these regions may form a cortical network for directed attention and orientation in space. A subsequent behavioral study confirmed that unilateral lesions restricted to the VLO produced significant multimodal neglect and extinction to bilateral simultaneous stimulation that recovered over the course of several weeks (King et al., 1989).

The first study that suggested that PPC lesions might produce multimodal neglect was done by Kirvel, Greenfield, and Meyer (1974). Their results indicated that very large posterior cortical lesions, which likely included the PPC, produced multimodal neglect to visual and tactile stimulation. A subsequent study with more restricted lesions found that unilateral destruction of the parietal cortex produced severe multimodal neglect of visual, tactile, auditory, and nociceptive stimuli presented to the contralesional side of the body (Crowne et al., 1986). The subjects recovered spontaneously over the course of several weeks. These results were strikingly similar to those obtained after unilateral lesions of the AGm, which produced multimodal neglect, allesthetic/allocentric responding, and spontaneous recovery that occurred over the course of several weeks (Corwin et al., 1986; Crowne \& Pathria, 1982; Crowne, Richardson, \& Ward, 1983).

Although the above two studies had examined the effects of PPC lesions on neglect, the nature of the lesions clouded the interpretations of these studies (Crowne et al., 1986; Kirvel et al., 1974). Although severe neglect was obtained in the Crowne et al. (1986) study, the lesions were limited to the parietal cortex rostral to what was later defined as the rodent PPC (Chandler et al., 1992; Kolb \& Walkey, 1987; Reep et al., 1994). The lesions in the Kirvel et al. study were very large and included direct damage to the primary visual and somatosensory cortices. Thus, unlike studies of the AGm and VLO, the issue of whether restricted lesions of the PPC would produce deficits in directed attention and orientation had not been unambiguously determined in rodents.

Two more recent studies directly examined the effects of relatively restricted unilateral lesions of the PPC, as defined by anatomical studies (Chandler et al., 1992; Kolb \& Walkey, 1987). Foreman, Save, Thinus-Blanc, and Buhot (1992) found that unilateral PPC lesions produced a neglect-like response to the distracting properties of ipsilesional and contralesionally flashing lights that were presented during the performance of a visual discrimination task. Ipsilesionally presented stimuli produced significant distraction and increased the latency to respond in the task; the identical stimulus presented contralesionally did not produce distraction. Further, when the distractors were presented simultaneously to both sides of the body, the animals demonstrated a strong tendency to respond to the ipsilesional distractor, as if the subjects were demonstrating extinction to bilateral simultaneous stimulation. Unlike the typical findings in studies of neglect, the subjects did demonstrate orientation to the contralesionally presented stimuli. King and Corwin (1993) examined the effects of unilateral destruction of the PPC, as defined by Kolb and Walkey, on orientation behavior to visual, tactile, and auditory stimuli. Their results indicated that unilateral lesions that are largely restricted to the PPC resulted in multimodal neglect of visual, tactile, and auditory stimulation and in significant allesthesia/allokinesia. These effects were quite similar to the results of Crowne et al. (1986), which were obtained with more rostral lesions of the parietal cortex. The results of these studies strongly support the contention that the area defined on anatomical grounds as the rodent PPC (Chandler et al., 1992; Kolb \& Walkey, 1987; Reep et al., 1994) is involved in directed attention. The issue of why the same results were found in the Crowne et al. study with more rostral lesions that did not directly damage the PPC remained to be determined.

\section{Comparisons Between Neglect \\ Induced by Lesions of the PPC or the AGm}

Neglect exhibits several common features, whether produced by PPC, AGm, or VLO lesions. In each instance, it is multimodal; there is spontaneous although incomplete recovery over 3-6 weeks; and there is significant allesthetic/allokinetic responding. In a series of recent studies from our laboratory, we examined the effects of various manipulations on recovery from neglect induced by lesions of the PPC and AGm and found a number of striking similarities. Acute behavioral recovery from severe neglect induced by unilateral destruction of either the PPC or the AGm can be produced by the administration of dopamine receptor agonists (Corwin, Burcham, \& Hix, 1996; Corwin et al., 1986; King \& Corwin, 1990; Vargo, Bromberg, Best, Corwin, \& Marshall, 1996). For both PPC and AGm operates, dopamine receptor agonists produced dose-dependent recovery from severe neglect. The recovery was present within 20 min of apomorphine administration, and testing on the following day revealed a reinstatement of severe neglect. Further, an environmental manipulation, $48 \mathrm{~h}$ of light deprivation, has been found to dramatically effect recovery from AGm- or PPC-induced neglect. A series of studies of the AGm has found that $48 \mathrm{~h}$ of light deprivation produces a virtually complete accelerated recovery from severe neglect (Corwin \& Burcham, 1995; Corwin \& Vargo, 1993; Crowne \& Pathria, 1982; Crowne et al., 1983). The recovery was found on the first post-light deprivation test and was maintained for the duration of behavioral testing. Burcham, Corwin, and Van Vleet (1998) examined the effects of light deprivation on PPC-induced neglect and found that light deprivation produced virtually identical effects on PPC operates. Light deprivation produced immediate recovery across all stimulus modalities, and the recovery was main- 
tained for the 3 weeks of behavioral testing. Thus, not only is the neglect induced by PPC or AGm lesions very similar, but also manipulations that influence behavioral recovery from neglect affect the PPC and AGm in a virtually identical manner.

In summary, studies of the effects of unilateral PPC lesions have revealed two major findings. First, the results of studies of the rodent PPC have found that, in rats, as in primates, unilateral destruction of the PPC produces severe neglect for visual, tactile, and auditory stimuli presented to the contralesional body side. As in primates, the subjects recover spontaneously over the course of weeks to months, and recovery is often incomplete. Further, acute recovery from severe PPC-induced neglect can be produced by the administration of dopamine receptor agonists, an effect obtained in humans with chronic neglect induced by fronto-parieto-temporal cortex damage (Fleet, Valenstein, Watson, \& Heilman, 1987). The neglect findings, together with the findings on spatial processing, support the qualitative similarity in PPC function between rodents and primates. A second issue of some importance is the finding that the anatomically interconnected PPC, AGm, and VLO are not only involved in spatial processing but also directed attention. These two disorders are clearly linked. Neglect in humans is often considered a hemispatial deficit. That is, not only is there a deficit in the responsiveness to externally applied stimulation, but there is also a disruption in the cognitive representation of space and in shifting spatial perspective (Bisiach \& Luzzati, 1978). These spatial and attentional deficits go hand-in-hand in rodents as well. Unilateral lesions of either the PPC, the AGm, or the VLO produce neglect, and bilateral destruction produces deficits in spatial processing. The behavioral findings, together with the anatomical findings, suggest that these three regions form a parallel distributed cortical system for directed spatial attention, as has been suggested for primates (Heilman et al., 1985; Mesulam, 1990; Selemon \& GoldmanRakic, 1988).

\section{TESTING THE CORTICAL NETWORK HYPOTHESIS}

The concept that frontal and parietal association areas function as an interdependent system was originally hypothesized for primates (Heilman et al., 1985; Mesulam, 1990) and is supported by PET studies in humans (Coull, Frith, Frackowiak, \& Grasby, 1996) but has not been tested experimentally. This is largely due to the fact that the axons interconnecting parietal and prefrontal areas travel in the white matter of the superior longitudinal fasciculus along with other axon systems, making them inaccessible to selective manipulation. In contrast, Vandevelde et al. (1996) found that, in rats, corticocortical axons travel in the deep gray matter and are segregated from other axonal systems that travel in the white matter, including thalamocortical axons, projections to subcortical regions from the cortex, and commissural fibers. This discovery presented the opportunity to test the hypothesis that the PPC, AGm, and VLO function together as a distributed cortical network for directed attention. We reasoned that, because of their longitudinal trajectories, the axons linking the PPC and AGm could be selectively transected by making a coronally oriented knife cut that extends into layer VI of the cortex, is located between the PPC and the AGm, and does not affect the white matter or cause significant damage to the cortical area in which the knife cut is made. If intact circuitry linking these three areas is required for normal directed attention, disruption of these connections in the absence of damage to any of the areas themselves should produce neglect.

Burcham, Corwin, et al. (1997) found that knife cuts like those described above produce neglect that is strikingly similar to that resulting from unilateral destruction of either the AGm or the PPC (Figure 3B). Following any of these procedures, subjects demonstrate significant contralesional multimodal neglect and allesthetic/allokinetic responding. Furthermore, the deficits produced by the knife-cut procedurc are as severe as those found after unilateral destruction of the AGm or PPC at an equivalent time postsurgery (Corwin et al., 1986; Corwin \& Vargo, 1993; Crowne et al., 1983; King \& Corwin, 1990, 1993; Vargo, Richard-Smith, \& Corwin, 1989). Control operates received a shallow knife cut in the same location that extended no deeper than layer V, and they exhibited no neglect (Figure 3B). After behavioral testing was completed, intracerebral injection of an anterograde axonal tracer (fluororuby) was used to verify that the disconnection was successful. Mapping the distribution of labeled axons revealed that the deeper knife cuts made in the experimental group had interrupted the axons traveling between the PPC and the AGm, whereas the shallower knife cuts made in the control group had not. The behavioral differences between the two groups were not due to disparities in lesion size, because there was no significant difference between the two groups in the amount of damage produced by the knife cuts. These results indicate that selective disconnection of the axons linking the PPC and AGm results in neglect that is indistinguishable from that produced by lesioning either area directly. Furthermore, these findings strongly support the contention that the PPC and AGm function as components of a distributed network that subserves directed attention and lend credence to the view that frontal-parietal circuitry is similarly organized in rodents and primates.

The knife-cut findings also have implications for the interpretation of prior behavioral studies utilizing electrolytic or other non-axon-sparing lesions. Such damage, even if restricted to the gray matter, necessarily interrupts numerous corticocortical axons, such as those interconnecting the PPC, AGm, and VLO. In the absence of axonal tracing in such cases, the true extent of the damage remains unknown, rendering a clear interpretation of behavioral findings difficult if not impossible. For example, the results of Crowne et al. (1986) demonstrated severe neglect after unilateral lesions that were too rostral to have 
damaged the PPC, raising the possibility that their lesions may have disconnected the PPC from the AGm and produced neglect as a result. Similarly, with regard to the role of the rodent PPC in spatial performance, we discussed in an earlier section that bilateral damage to the rostral parietal cortex often produces spatial deficits that are virtually equivalent to those obtained after lesions that directly damage the PPC (Kesner et al., 1989; Save et al., 1992; Thinus-Blanc et al., 1996). These results suggest that deficits in allocentric tasks produced by the more rostral parietal lesions may have been the result of bilateral disconnections of the PPC from the VLO, which is also involved in allocentric spatial processing (Corwin, Fussinger, Meyer, King, \& Reep, 1994).

\section{SUMMARY AND CONCLUSIONS}

The PPC in rodents has been found to be a region that receives multimodal corticocortical inputs, and it is strongly interconnected with two other multimodal regions, the AGm and the VLO. Further, all of these regions have been found to be involved in spatial processing and directed attention. The behavioral effects of disconnection of the PPC from these prefrontal regions appear to be virtually identical to the effects of direct destruction of the PPC. Unilateral lesions of the PPC, the AGm, or the VLO or disconnections of the PPC from the AGm and the VLO produce neglect, and bilateral destruction of any of these areas produces deficits in spatial processing. The perspective of the present review has been that the deficits produced by PPC lesions are best interpreted by viewing the PPC, together with the AGm and VLO, as a distributed cortical network for spatial processing and directed attention, as has been hypothesized for primates (Heilman et al., 1985; Mesulam, 1990; Selemon \& Goldman-Rakic, 1988). This perspective is in accord with a number of theoretical and applied reviews that have stressed the importance of frontal-parietal interactions for complex cognitive processes, including spatial processing (Selemon \& Goldman-Rakic, 1988), attention (Heilman et al., 1985; Mesulam, 1990), and a thought disorder typical of one type of schizophrenia (Buchanan et al., 1993).

Several recent studies in primates suggest that the PPC integrates information into a spatial representation that is used to guide movements. This integration involves combining multimodal sensory information with motorrelated information generated in the frontal cortex, and these frontal-parietal interactions are envisioned to function as an integrated, dynamic network that continuously evaluates and updates body position and movement relative to external space (Andersen, Snyder, Bradley, \& Xing, 1997; Wise, Boussaoud, Johnson, \& Caminiti, 1997). Therefore, directed attention is considered to be an emergent property of the network as a whole rather than the sum of individual components, such as perception, motivation, and exploration (Mesulam, 1990). Consistent with this view, a key feature of this and other networks is the absence of a one-to-one correspondence between single anatomical sites and behavior (Mesulam, 1990). This would explain why the cortical disconnections we performed in rats produced full-blown neglect in the absence of significant damage to the cortical areas themselves.

Rodents possess much less differentiated frontal and parietal cortices than do primates, rendering it difficult to make direct comparisons among specific cortical areas. Nevertheless, it appears that large-scale frontal-parietal interactions are common to both groups, and we suspect that they are a common feature of mammalian brains. Although we have emphasized that the rodent PPC is one component of a network also involving the $\mathrm{AGm}$ and VLO, further studies are needed to better understand the computations made by each of these areas and the interactions among them that result in the complex behavior exemplified by directed attention in space.

\section{REFERENCES}

Andersen, R. A., Snyder, L. H., Bradley, K. C., \& Xing, J. (1997). Multimodal representation of space in the posterior parietal cortex and its use in planning movements. Annual Review of Neuroscience, 20, 303-330.

ARNAulT, P., \& Roger, M. (1990). Ventral temporal cortex in the rat: Connections of secondary auditory areas $\mathrm{Te} 2$ and Te3. Journal of Comparative Neurology, 302, 110-123.

BisiaCH, E., \& LuZzaTI, C. (1978). Unilateral neglect of representational space. Cortex, 14, 129-133.

Buchanan, R. W., Breier, A., Kirkpatrick, B., Elkashef, A., Munson, R. C., Gellad, F., \& Carpenter, W. T. (1993). Structural abnormalities in deficit and nondeficit schizophrenia. American Journal of Psychiatry, 150, 59-65.

BurchaM, K. J., Corwin, J. V., \& ReEP, R. L. (1997). Long-term effects of disconnection of medial granular and posterior parietal cortex in rats. Society for Neuroscience Abstracts, 23, 230.

Burcham, K. J., Corwin, J. V., Stoll, M. L., \& ReEP, R. L. (1997). Disconnection of medial granular and posterior parietal cortex produces multimodal neglect in rats. Behavioural Brain Research, 86, 41-47.

Burcham, K. J., Corwin, J. V., \& Van Vleet, T. M. (1998). Light deprivation produces a therapeutic effect on neglect induced by unilateral destruction of the posterior parietal cortex in rats. Behavioural Brain Research, 90, 187-197.

Cavada, C., \& Goldman-Rakic, P. S. (1989a). Posterior parietal cortex in thesus monkey: I. Parcellation of areas based on distinctive limbic and sensory corticocortical connections. Journal of Comparative Neurology, 287, 393-421.

Cavada, C., \& Goldman-Rakic, P. S. (1989b). Posterior parietal cortex in rhesus monkey: II. Evidence for segregated corticortical networks linking sensory and limbic areas with the frontal lobe. Journal of Comparative Neurology, 287, 422-445.

Caviness, V. S. (1975). Architectonic map of neocortex of the normal mouse. Journal of Comparative Neurology, 164, 247-264.

Chandler, H. C., King, V., Corwin, J. V., \& ReEP, R. L. (1992). Thalamocortical connections of rat posterior parietal cortex. Neuroscience Letters, 143, 237-242.

Chen, L. L., Lin, L.-H., Green, E. J., Barnes, C.A., \& MCNaughton, B. L. (1994). Head-direction cells in the rat posterior cortex: I. Anatomical distribution and behavioral modulation. Experimental Brain Research, 101, 8-23.

Chen, L. L., \& McNaughton, B. L. (1988). Spatially selective discharge of vision and movement modulated posterior parietal neurons in the rat. Society for Neuroscience Abstracts, 14, 818.

Corwin, J. V., \& BurchaM, K. J. (1995). Temporal factors related to the therapeutic effect of light deprivation on multimodal neglect, in rats. Society for Neuroscience Abstracts, 21, 175.

Corwin, J. V., Burcham, K. J., \& Hix, G. I. (1996). Apomorphine pro- 
duced an acute dose-dependent therapeutic effect on neglect produced by unilateral destruction of the posterior parietal cortex in rats. Behavioural Brain Research, 79, 41-49.

CoRwin, J. V., Fussinger, M., Meyer, R. M., King, V. R., \& ReeP, R. L (1994). Bilateral destruction of the ventrolateral orbital cortex produces allocentric, but not egocentric spatial deficits in rats. Behavioural Brain Research, 61, 79-86.

Corwin, J. V., Kanter, S., Watson, R. T., Heilman, K. M., ValenSTEIN, E., \& HASHIMOTo, A. (1986). Apomorphine has a therapeutic effect on neglect produced by unilateral dorsomedial prefrontal cortex lesions in rats. Experimental Neurology, 94, 683-689.

CoRWIN, J. V., \& VARGO, J. M. (1993). Light deprivation produces accelerated behavioral recovery of function from neglect produced by unilateral medial agranular prefrontal cortex lesions in rats. Behavioural Brain Research, 56, 187-196.

Coull, J. T., Frith, C. D., Frackowiak, R. S. J., \& Grasby, P. M. (1996). A fronto-parietal network for rapid visual information processing: A PET study of sustained attention and working memory. Neuropsychologia, 34, 1085-1095.

COWEY, A., \& BOZEK, T. (1974). Contralateral "neglect" after unilateral dorsomedial prefrontal lesions in rats. Brain Research, 72, 53-63.

Crowne, D. P. (1983). The frontal eye field and attention. Psychological Bulletin, 93, 232-260.

Crowne, D. P., Novotny, M. F., MaIER, S. E., \& Vitols, S. R. (1992). Effects of unilateral parietal lesions on spatial localization in the rat. Behavioral Neuroscience, 106, 811-822.

Crowne, D. P., \& Pathria, M. N. (1982). Some attentional effects of unilateral frontal lesions in the rat. Behavioural Brain Research, 6 , 25-32.

Crowne, D. P., Richardson, C. M., \& Dawson, K. A. (1986). Parietal and frontal eye field neglect in the rat. Behavioural Brain Research, 22, 227-231.

Crowne, D. P., Richardson, C. M., \& Ward, G. (1983). Brief deprivation of vision after unilateral lesions of the frontal eye fields prevents contralateral inattention. Science, 220, 527-530.

DeueL, R. K. (1987). Neural dysfunction during hemineglect after cortical damage in two monkey models. In M. Jeannerod (Ed.), Neurophysiological and neuropsychological aspects of spatial neglect (pp. 315-334). Amsterdam: Elsevier, North-Holland.

Di, S., BretT, B., \& BarTH, D. S. (1994). Polysensory evoked potentials in rat parietotemporal cortex: Combined auditory and somatosensory responses. Brain Research, 642, 267-280.

DiMATTIA, B. V., \& KESNER, R. P. (1988a). Role of the posterior parietal association cortex in the processing of spatial event information. Behavioral Neuroscience, 102, 397-403.

DiMatTIA, B. V., \& KeSNer, R. P. (1988b). Spatial cognitive maps: Differential role of parietal cortex and hippocampal formation. Behavioral Neuroscience, 102, 471-480.

Donoghue, J. P., Kerman, K. L., \& Ebner, F. F. (1979). Evidence for two organizational plans within the somatic sensory-motor cortex of the rat. Journal of Comparative Neurology, 183, 647-664.

Donoghue, J. P., \& WISE, S. P. (1982). The motor cortex of the rat: Cytoarchitecture and microstimulation mapping. Journal of Comparative Neurology, 212, 76-88.

FABRI, M., \& BURTON, H. (1991). Ipsilateral cortical connections of primary somatic sensory cortex in rats. Journal of Comparative Neurology, 311, 405-424.

Fleet, W. S., Valenstein, E., Watson, R. T., \& Heilman, K. M. (1987). Dopamine agonist therapy for neglect in humans. Neurology, 37, 1765-1770.

Foreman, N., Save, E., Thinus-Blanc, C., \& Buhot, M.-C. (1992). Visually guided locomotion, distractibility, and the missing-stimulus effect in hooded rats with unilateral or bilateral lesions. Behavioral Neuroscience, 106, 529-538.

GIOANNI, Y., \& LAMARCHE, M. (1985). A reappraisal of rat motor cortex organization by intracortical microstimulation. Brain Research, $344,49-61$

Goldman-Rakic, P. S. (1988). Topography of cognition: Parallel distributed networks in primate association cortex. Annual Review of Neuroscience, 11, 137-156.
HALL, R. D., \& Lindholm, E. P. (1974). Organization of motor and somatosensory neocortex in the albino rat. Brain Research, 66, 23-38.

Harting, J. K., Hall, W. C., \& Diamond, I. T. (1972). Evolution of the pulvinar. Brain, Behavior \& Evolution, 6, 424-452.

Heilman, K. M., Watson, R. T., \& Valenstein, E. (1985). Neglect and related disorders. In K. M. Heilman \& E. Valenstein (Eds.), Clinical neuropsychology (2nd ed., pp. 243-293). New York: Oxford University Press.

Heilman, K. M., Watson, R. T., \& Valenstein, E. (1993). Neglect and related disorders. In K. M. Heilman \& E. Valenstein (Eds.), Clinical neuropsychology (3rd ed., pp. 279-336). New York: Oxford University Press.

Herrmann, T., Poucet, B., \& Ellen, P. (1985). Spatial problem solving in a dual runway task by normal and septal rats. Behavioral Neuroscience, 99, 631-637.

JoNES, E. G., \& LEAVITT, R. Y. (1974). Retrograde axonal transport and the demonstration of non-specific projections to the cerebral cortex and striatum from thalamic intralaminar nuclei in the rat, cat and monkey. Journal of Comparative Neurology, 154, 349-378.

Kesner, R. P., Farnsworth, G., \& DimatTia, B. V. (1989). Double dissociation of egocentric and allocentric space following prefrontal and parietal cortex lesions in the rat. Behavioral Neuroscience, 103, 956-961.

KING, V., \& CoRwIN, J. V. (1990). Neglect following unilateral ablation of the caudal but not the rostral portion of medial agranular cortex of the rat and the therapeutic effect of apomorphine. Behavioural Brain Research, 37, 169-184.

KING, V., \& CORWIN, J. V. (1992) . Spatial deficits and hemispheric asymmetries in the rat following unilateral and bilateral lesions of posterior parietal or medial agranular cortex. Behavioural Brain Research, 50, 53-68.

KING, V., \& CoRWIN, J. V. (1993). Comparison of hemi-inattention produced by unilateral lesions of the posterior parietal cortex or medial agranular prefrontal cortex in rats: Neglect, extinction, and the role of stimulus distance. Behavioural Brain Research, 54, 117-131.

KING, V., CoRwin, J. V., \& ReEP, R. L. (1989). Production and characterization of neglect in rats with unilateral lesions of ventrolateral orbital cortex. Experimental Neurology, 105, 287-299.

KiRVEL, R. D., GREenfield, R. A., \& MEYER, D. R. (1974). Multimodal sensory neglect in rats with radical unilateral posterior isocortical and superior collicular ablations. Journal of Comparative \& Physiological Psychology, 87, 156-162.

KoLB, B. (1984). Functions of the frontal cortex in the rat. Brain Research Reviews, 8, 65-98.

Kolb, B., Buhrmann, K., McDonald, R., \& Sutherland, R. J. (1994). Dissociation of the medial prefrontal, posterior parietal, and posterior temporal cortex for spatial navigation and recognition memory in the rat. Cerebral Cortex, 4, 664-680.

Kolb, B., Nonneman, A. J., \& Singh, R. K. (1974), Double dissociation of spatial impariments and perseveration following selective prefrontal lesions. Journal of Comparative \& Physiological Psychology, 87, 772-780.

Kolb, B., Sutherland, R. J., \& Whishaw, I. Q. (1983). A comparison of the contributions of the frontal and parietal association cortex to spatial localization in rats. Behavioural Neuroscience, 97, 13-27.

KoLB, B., \& WALKEY, J. (1987). Behavioural and anatomical studies of the posterior parietal cortex in the rat. Behavioural Brain Research, 23, 127-145

Koralek, K. A., Olavarria, J., \& Killackey, H. P. (1990). Areal and laminar organization of corticocortical projections in the rat somatosensory cortex. Journal of Comparative Neurology, 299, 133-150.

KRETTEK, J. E., \& PRICE, J. L. (1977). The cortical projections of the mediodorsal nucleus and adjacent thalamic nuclei in the rat. Journal of Comparative Neurology, 71, 157-192.

KRIEG, W. J. S. (1946). Connections of the cerebral cortex: I. Albino rat: A. Topography of the cortical areas. Journal of Comparative Neurology, 84, 221-275.

LeichnetZ, G. R., \& GonZalo-Ruiz, A. (1987). Collateralization of frontal eye field (medial precentral/anterior cingulate) neurons projecting to the paraoculomotor region, superior colliculus, and medial 
pontine reticular formation in the rat: A fluorescent double-labeling study. Experimental Brain Research, 68, 355-364.

Leichnetz, G. R., Hardy, S. G. P., \& CarRuth, M. K. (1987). Frontal projections to the region of the oculomotor complex in the rat: A retrograde and anterograde HRP study. Journal of Comparative Neurology, 263, 387-399.

LEONARD, C. M. (1969). The prefrontal cortex of the rat: I. Cortical projection of the mediodorsal nucleus. II. Efferent connections. Brain Research, 12, 321-343.

LEONARD, C. M. (1972). The connections of the dorsomedial nuclei. Brain, Behavior \& Evolution, 6, 524-541.

Li, X.-G., Florence, S. L., \& KaAs, J. H. (1990). Areal distributions of cortical neurons projecting to different levels of the caudal brain stem and spinal cord in rats. Somatosensory \& Motor Research, 7, 315-335.

McDaniel, W. F., Via, J. D., Smith, J. S., Wells, D. L., Fu, J. J., BisHop, J. F., BOYD, P. A., \& LEDESMA, H. M. (1995). Unilateral injury of posterior parietal cortex and spatial learning in hooded rats. Behavioural Brain Research, 70, 165-179.

McNaughton, B. L., Leonard, B., \& Chen, L. (1989). Corticalhippocampal interactions and cognitive mapping: $\mathrm{A}$ hypothesis based on reintegration of the parietal and inferotemporal pathways for visual processing. Psychobiology, 17, 236-246.

Mesulam, M.-M. (1990). Large-scale neurocognitive networks and distributed processing for attention, language, and memory. Annals of Neurology, 28, 597-613.

MiLlER, M. W. (1987). The origin of corticospinal projection neurons in rat. Experimental Brain Research, 67, 339-351.

Miller, M. W., \& VoGt, B. A. (1984). Direct connections of rat visual cortex with sensory, motor, and association cortices. Journal of Comparative Neurology, 226, 184-202.

Morris, R. M., Garrud, P., Rawlins, J. N. P., \& O'Keefe, J. (1982). Place navigation impaired in rats with hippocampal lesions. Nature, 297, 681-683.

NeAfsey, E. J., Bold, E. L., HAas, G., Hurley-Guis, K. M., Quirk, G., SiEverT, C. F., \& TERrEBERRY, R. R. (1986). The organization of the rat motor cortex: A microstimulation mapping study. Brain Research Reviews, 11, 77-96.

Neafsey, E. J., \& Sievert, C. (1982). A second forelimb motor area exists in rat frontal cortex. Brain Research, 232, 151-156.

Nonneman, A. J., Voigt, J., \& KolB, B. E. (1974). Comparisons of behavioral effects of hippocampal and prefrontal cortex lesions in the rat. Journal of Comparative \& Physiological Psychology, 87, 249-260.

Olton, D. (1977). Spatial memory. Scientific American, 236, 82-99.

Paterson, A., \& Zangwill, O. L. (1944). Disorders of visual space perception associated with lesions of the right cerebral hemisphere. Brain, 67, 331-358.

Paxinos, G., \& WaTSON, C. (1986). The rat brain in stereotaxic coordinates (2nd ed.). New York: Academic Press

PoHL, W. (1973). Dissociation of spatial discrimination deficits following frontal and parietal lesions in monkeys. Journal of Comparative \& Physiological Psychology, 82, 227-239.

ReEP, R. L., Chandler, H. C., \& CoRWIN, J. V. (1994). Rat posterior parietal cortex: Topography of cortico-cortical and thalamic connections. Experimental Brain Research, 100, 67-84.

ReEP, R. L., CoRWIN, J. V., HASHIMOTO, A., \& Watson, R. T. (1984). Afferent connections of medial precentral cortex in the rat. Neuroscience Letters, 44, 247-252.

Reep, R. L., CoRwin, J. V., Hashimoto, A., \& Watson, R. T. (1987). Efferent connections of the rostral portion of medial agranular cortex in rats. Brain Research Bulletin, 19, 203-221.

REEP, R. L., Corwin, J. V., \& King, V. (1996). Neuronal connections of orbital cortex in rats: Topography of cortical and thalamic afferents. Experimental Brain Research, 111, 215-232.

REEP, R. L., \& GoODWIN, G. S. (1988). Layer VII of rodent cerebral cortex. Neuroscience Letters, 90, 15-20.

REEP, R. L., Goodwin, G. S., \& CoRwin, J. V. (1990). Topographic organization in the corticocortical connections of medial agranular cortex in rats. Journal of Comparative Neurology, 294, 262-280.

Roger, M., \& ArNault, P. (1989). Anatomical study of the connec- tions of the primary auditory area in the rat. Journal of Comparative Neurology, 287, 339-356.

RomanSKI, L. M., \& LeDoux, J. E. (1993). Organization of rodent auditory cortex: Anterograde transport of Pha-L from MGv to temporal neocortex. Cortex, 3, 499-514.

Rose, M. (1912). Histologische Lokalisation der Grosshirnrinde bei kleinen Saugetieren (Rodentia, Insectivora, Chiroptera) [Histological localization of cerebral cortex among small mammals (Rodentia, Insectivora, Chiroptera)]. Journal of Psychology \& Neurology, 19, 391-479.

Sanderson, K. J., Welker, W., \& Shambes, G. M. (1984). Reevaluation of motor cortex and of sensorimotor overlap in cerebral cortex of albino rats. Brain Research, 292, 251-260.

SAVE, E., \& MoghadDam, M. (1996). Effects of lesions of the associative parietal cortex on the acquisition and use of spatial memory in egocentric and allocentric navigation tasks in the rat. Behavioral Neuroscience, 110, 74-85.

Save, E., Poucet, B., Foreman, N., \& Buhot, M.-C. (1992). Object exploration and reactions to spatial and nonspatial changes in hooded rats following damage to parietal cortex or hippocampal formation. Behavioral Neuroscience, 106, 447-456.

SchmahmanN, J. D., \& Pandya, D. N. (1990). Anatomical investigation of projections from thalamus to posterior parietal cortex in the rhesus monkey: A WGA-HRP and fluorescent tracer study. Journal of Comparative Neurology, 295, 299-326.

Schober, W., \& Winkelmann, E. (1975). Der visuelle Kortex der Ratte Cytoarchetectonik und stereotaktische Parameter [The visual cortex of the rat-cytoarchetectonic and stereotaxic parameters]. Zeitschrift für Mikroskopik Anatomie Forschung, 89, 431-446.

Selemon, L. D., \& Goldman-Rakic, P. S. (1988). Common cortical and subcortical targets of the dorsolateral prefrontal and posterior parietal cortices in the rhesus monkey: Evidence for a distributed neural network subserving spatially guided behavior. Journal of Neuroscience, 8, 4049-4068.

Semmes, J., Weinstein, S., Ghent, L., \& Teuber, H. (1963). Correlates of impaired orientation. Brain, 86, 747-772.

Sinnamon, H. M., \& Charman, C. S. (1988). Unilateral and bilateral lesions of the anteromedial cortex increase perseverative head movements of the rat. Behavioural Brain Research, 27, 145-160.

SinNAMON, H. M., \& GALER, B. S. (1984). Head movements elicited by electrical stimulation of the anteromedial cortex of the rat. Physiology \& Behavior, 33, 185-190.

Sugishita, M., EtTlinger, G., \& Ridley, R. M. (1978). Disturbance of cage finding in the monkey. Cortex, 14, 431-438.

SUKEKAWA, K. (1988). Interconnections of the visual cortex with the frontal cortex in the rat. Journal für Hirnforschung, 29, 83-93.

Thinus-Blanc, C., Save, E., Poucet, B., \& Foreman, N. (1996). Effects of parietal cortex lesions on spatial problem solving in the rat. Behavioural Brain Research, 81, 115-121.

Torrealba, F., Olavarria, J., \& Carrasco, M. A. (1984). Cortical connections of the anteromedial extrastriate visual cortex in the rat. Experimental Brain Research, 56, 543-549.

VALLAR, G. (1993). The anatomical basis of spatial hemineglect in humans. In I. H. Robertson \& J. C. Marshall (Eds.), Unilateral neglect: Clinical and experimental studies (pp. 27-59). Hillsdale, NJ: Erlbaum.

VANDevelde, I. L., DuckworTh, E., \& ReEP, R. L. (1996). Layer VII and the gray matter trajectory of corticocortical axons in rats. Anatomy \& Embryology, 194, 581-593.

VAN Groen, T., \& Wyss, J. J. (1992). Connections of the retrosplenial dysgranular cortex in the rat. Journal of Comparative Neurology, 315, 200-216

Vargo, J. M., Bromberg, B. B., Best, P. J., Corwin, J. V., \& Marshall, J. F. (1996). D1-class dopamine receptor involvement in the behavioral recovery from prefrontal cortical injury. Behavioural Brain Research, 72, 39-48.

VARGo, J. M., CoRWIN, J. V., KING, V., \& ReEP, R. L. (1988). Differential behavioral effects of left and right hemisphere lesions of medial prefrontal cortex in rats. Experimental Neurology, 102, 199-209.

VARGo, J. M., Richard-Smith, M., \& CoRwin, J. V. (1989). Spiroperi- 
dol reinstates asymmetries in neglect in rats recovered from left or right dorsomedial prefrontal cortex lesions. Behavioral Neuroscience, 103, 1017-1027.

VoGt B. A., \& Miller M. W. (1983). Cortical connections between rat cingulate cortex and visual, motor, and postsubicular cortices. Journal of Comparative Neurology, 216, 192-210.

Wise, S. P., Boussaoud, D., Johnson, P. V., \& Caminiti, R. (1997). Premotor and parietal cortex: Corticocortical connectivity and combinatorial computations. Annual Review of Neuroscience, 20, 25-42.

ZILLES, K. (1985). The cortex of the rat: A stereotaxic atlas. Berlin: Springer-Verlag.

ZilLES, K., \& WREE, A. (1985). Cortex: Areal and laminar structure. In
G. Paxinos (Ed.), The rat nervous system: Forebrain and midbrain (Vol. 1, pp. 375-415). Sydney: Academic Press.

ZiLles, K., WREE, A., \& DAUSCH, N.-D. (1990). Anatomy of the neocortex: Neurochemical organization. In B. Kolb \& R. C. Tees (Eds.), The cerebral cortex of the rat (pp. 113-150). Cambridge, MA: MIT Press. Zilles, K., Zilles, B., \& SCHleicher, A. (1980). A quantitative approach to cytoarchitectonics: VI. The areal pattern of the cortex of the albino rat. Anatomy \& Embryology, 159, 335-360.

(Manuscript received August 4, 1997; revision accepted for publication October 20,1997.) 